\title{
Formación de profesores de biología en tiempos de pandemia. ¿Qué tecnologías se usaron para la educación remota de emergencia?
}

\section{Training of biology teachers in times of pandemic. Which technologies were used for remote emergency education?}

\author{
JAVIER GRILLI SILVA ${ }^{1}$ \\ ORCID: 0000-0002-6690-1659 \\ javier.grilli@gmail.com \\ DARÍO DALMÁS ${ }^{1}$ \\ ORCID: 0000-0002-3157-3835 \\ dadalm22@gmail.com
}

ANDREA PRADO
ORCID: 0000-0002-6071-0982
avpn0806@gmail.com
Recibido: 25 de febrero de 2021 - Aceptado: 18 de mayo de 2021

\section{Resumen}

El artículo presenta resultados de una investigación de tipo cuantitativa/cualitativa, realizada en un Centro de formación de profesores de Uruguay, sobre la educación remota a distancia que devino por la emergencia sanitaria decretada a raíz de la pandemia por la Covid-19. Concretamente el artículo presenta resultados sobre el perfil socio-educativo de los estudiantes de profesorado de Biología y sobre los recursos tecnológicos que se utilizaron para los procesos de enseñanza-aprendizaje, en las asignaturas específicas de este profesorado. Se confirman tendencias constatadas por otros estudios: la mayoría de nuestro estudiantado tiene una edad por encima de lo esperable, es decir, sin rezago (tanto en el ingreso como en el egreso de la carrera), hay una feminización marcada y el estudiante está cursando asignaturas específicas de varios niveles de profesorado. Con respecto al uso que se hizo de las tecnologías para llevar adelante los cursos del año lectivo 2020, se constata que

1 Centro Regional de Profesores del Litoral, Departamento de Biología, Salto, Uruguay tanto profesores como estudiantes utilizaron principalmente laptop personales y conexión a Internet por Wifi particular. Las laptops XO y la conectividad Ceibal ocupó un papel secundario, sin perjuicio de algunas diferencias observables entre los diferentes niveles de la carrera.

Palabras clave: Formación docente, educación a distancia, pandemia, plataformas educativas

\section{Abstract}

This article shows the results of a cualitative and cuantitative research, carried out by a Teacher Training Center from Uruguay, on remote education as a result of the present health emergency cause by the Covid 19 Pandemic. The article specifically shows results on the social-cultural profile of Biology Trainee Teachers and on the technological resources used by them for achieving the teaching 
- learning process in the specific subjects of this area. Trends observed by others studies are confirmed, most of our students are older than expected, that is, without lag (not only at the begining but also at the ending of the career), there is a strong tendency to feminisation, and students take specific subjects from different levels of the career. Concerning to the students' use of technology to continue with the 2020 courses, evidence shows that students and Teachers mainly used personal computers and Internet connection. Both, XO computers and Ceibal connection took second place in this process, despite of some observable differences between the different teaching Training levels.

Keywords: Teaching training, distance education, pandemic, educational platforms.

\section{Introducción}

En Uruguay el día viernes 13 de marzo del año 2020, se confirmaron los primeros 4 casos de Covid-19 y el gobierno, que había asumido el $1^{\circ}$ de marzo, decretó la emergencia sanitaria. Bajo esta medida se suspendieron las clases presenciales (que habían comenzado dos semanas atrás), en todo el sistema educativo del país, público y privado. Por esto, cuando el lunes 16 de marzo se retomaron las clases, fue en modalidad a distancia para todos los niveles de la Educación: primaria, secundaria, técnico-profesional, formación docente y Universidad.

Los institutos de formación docente de todo el país se sumaron, como lo hizo todo el sistema educativo no solo del país sino del continente y gran parte del mundo, a sostener en el contexto de una pandemia, la actividad curricular prevista para el año lectivo 2020. Esta situación de excepción tensó al máximo las capacidades institucionales, de los estudiantes y de los docentes, ya que hubo que re-adecuar, rápidamente, la enseñanza terciaria a un funcionamiento a distancia y mediado por la tecnología. Como lo plantea Aragay (2020), en este contexto la educación que se brinda debería llamarse remota de emergencia, pues abruptamente docentes y estudiantes han sido sumergidos en una modalidad a distancia, siendo parte de un sistema que no estaba pensado para ello. A diferencia de la concepción de educación a distancia, la enseñanza remota de emergencia es visualizada como una situación transitoria que puede ser revertida y a la vez como una oportunidad para incorporar algunos aprendizajes que potencien cambios educativos en un futuro (ANEP,
2020). Todo un sistema educativo pensado para la presencialidad, tuvo que adaptarse por circunstancias ajenas y forzosas, a una enseñanza-aprendizaje a distancia y mediadas por tecnología, la cual analizamos - para profesorado- en este trabajo.

Este artículo analiza la educación brindada en la formación de profesores pública de Uruguay, en el año lectivo 2020. El país tiene organizado su formación docente pública en un sistema no universitario que está bajo la dirección de un desconcentrado, el Consejo de Formación en Educación (CFE), dependiente del Consejo Directivo Central (CODICEN), bajo un Sistema Único de Formación Docente (SUNFD), conocido como Plan 2008. Del CFE dependen 34 Institutos de Formación de Docente, algunos ubicados en la capital del país y otros en el interior. De estos, algunos trabajan en la formación de docentes para el sistema primario (maestros), otros forman docentes para el sistema medio (profesores y maestros técnicos) y otros combinan ambas formaciones.

La investigación que presentamos se realizó en un Centro de Formación docente del interior del país que se dedica a la formación inicial y permanente de profesores en una región del territorio.

\section{Marco teórico}

Las tecnologías de la información y la comunicación (TIC) son no solamente un potente recurso para estar informados y comunicados en los tiempos actuales, sino también generadoras de un nuevo espacio socialeducativo: la educación virtual (Echeverría, 2002). Al pensarla en términos sistémicos, según Moore y Kearsley (2005), la educación virtual es el aprendizaje que tiene lugar mediante procesos educativos intencionales, en contextos de enseñanza mediados tecnológicamente, requiriendo el diseño específico de materiales de aprendizaje, el empleo orientado de diferentes herramientas de comunicación, así como arreglos organizacionales y administrativos que hacen posible su gestión. La educación virtual es por lo tanto una educación a distancia mediada por tecnologías digitales.

Las ventajas o beneficios de la educación virtual en el nivel terciario, son bastante conocidos: al posibilitar un manejo flexible de los tiempos para la realización de las actividades educativas y suprimir el desplazamiento físico para participar en ellas, permite compatibilizar la actividad laboral y familiar del educando con su formación, promueve una mayor autonomía e independencia del aprendiz, facilita el fácil acceso a 
recursos educativos en formatos digitales, entre otros beneficios. También la educación virtual trae aparejada importantes desafíos y exigencias, tanto para la institución educativa que lo administra, como para los docentes y los estudiantes. Algunos de ellos son: requerimiento de infraestructura tecnológica para mediar entre profesor-estudiante, capacitación de profesores para el buen desempeño de las funciones de tutor en línea, el replanteamiento de la dimensión individual y colectiva en los procesos de enseñanza y aprendizaje, evaluación de aprendizajes en contextos no presenciales. (Arranz, Aguado y Lucía, 2008; Inciarte Rodríguez, 2008; Yot y Marcelo, 2013; Pascoal, 2016; Ángel Rueda, Valdés Godínesa y Guzmán Flores, 2017).

En la educación a distancia mediada por tecnología, existen 3 grandes tendencias: el e-learning, el m-learning y el $b$-learning. El primero es el aprendizaje en línea, modelo de enseñanza y aprendizaje que emplea Internet y las TIC como herramientas para que los participantes puedan comunicarse e interactuar. En el mobile learning (m-learning), el aprendizaje se da a través de dispositivos móviles (teléfonos inteligentes laptop y tablets) que hacen posible aprender en cualquier lugar y en cualquier momento. Puede considerarse como la evolución del e-learning, pues ha supuesto un salto importante en el avance hacia la ubicuidad de la formación. El blended learning (b-learning), es el aprendizaje híbrido, es decir el que se da combinando entornos físicos con entornos virtuales, existiendo por tanto instancias de enseñanza y aprendizaje presenciales y no presenciales (Hidalgo, Orozco y Daza, 2015).

En cualquiera de las 3 variantes de la educación a distancia mediada por tecnología, se configuran espacios virtuales en los cuales se enseña y se aprende: las aulas virtuales. Área, San Nicolás y Sanabria (2018) definen las aulas virtuales como un lugar acotado reconocible en el ciberespacio que posee una identidad y estructura definida con fines educativos (página 180). Es un entorno virtual creado con intencionalidad pedagógica de estimular, guiar o supervisar un proceso de aprendizaje de modo formalizado. Son 4 los componentes básicos que deben contener las aulas virtuales: las actividades o tareas, los materiales didácticos u objetos de aprendizaje digitales, los procesos comunicativos y la tutorización evaluativa. (Area et al., 2018).

Ubicadas en plataformas digitales, educativas, las aulas virtuales permiten gestionar todas las actividades propias de la enseñanza y aprendizaje de una asignatura o curso. Facilitan el anclaje de los contenidos de aprendizaje en diversos formatos digitales, posibilitando las comunicaciones verticales y horizontales tanto síncronas como asíncronas, entre estudiantes y docente. Las plataformas educativas son por tanto una estructura tecnológica que responde a las exigencias de un determinado diseño pedagógico, estando ambos perfectamente integrados (García Aretio, 2014).

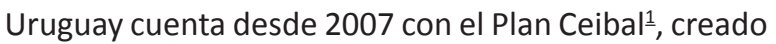
con la finalidad de promover la inclusión e igualdad de oportunidades educativas, apoyando con tecnologías, programas, recursos educativos y capacitación docente a todo el sistema educativo público. Desde su implementación cada estudiante que ingresa al sistema educativo en todo el país, en los niveles de primaria y media, accede a una computadora (conocida coloquialmente como "ceibalita" o XO) para su uso personal con conexión a Internet gratuita desde el centro educativo. En formación docente también los estudiantes acceden a la computadora del Plan Ceibal, en el $3^{\text {er }}$ año de la carrera. El Plan ofrece también la plataforma virtual Schoology-CREA 2, existiendo un distrito para el subsistema formación docente: CREA-CFE.

¿Cuán importantes son las XO y la conectividad de Ceibal para los estudiantes de profesorado? El propio CFE cuenta con algunas investigaciones realizadas que abordan estas preguntas (Conde, González y Villagrán, 2018; Rodríguez y Grilli, 2018). También hay otras investigaciones realizadas en la órbita privada que pusieron foco en el uso de dispositivos móviles en la formación de profesores (Vanden Berg, 2015; Rodríguez, Yot, Cabrera, Zorrilla, y Grilli, 2019). Como explicaremos a continuación, la presente investigación realizada por el equipo tiene también un objetivo en el uso de las tecnologías asociadas al Plan Ceibal, para la educación remota de emergencia que se dio en la formación de profesores en el año lectivo 2020. Como veremos más adelante, nuestros resultados y conclusiones son coincidentes en varios aspectos a las de los trabajos precedentemente citados.

Teniendo un objetivo general de la investigación ("caracterizar la enseñanza y el aprendizaje a distancia que se dio en las asignaturas específicas de profesorado de Biología durante el año lectivo 2020"), se apuntó a 3 objetivos específicos. En este artículo se presentan resultados del primero de ellos: determinar los recursos tecnológicos que se utilizaron para los procesos de enseñanzaaprendizaje, en las distintas asignaturas específicas del profesorado de Biología y para el Centro de Formación docente que se hace el relevamiento de datos.

1 https://www.ceibal.edu.uy/es/institucional 
En torno a este objetivo, las preguntas que guiaron el trabajo fueron: ¿cómo se dio la formación de profesores de Ciencias Biológicas en este contexto de educación a distancia?, ¿qué recursos tecnológicos primaron para el transcurso de las clases?, ¿qué dispositivos informáticos y qué tipo de conectividad se utilizó para mantener el contacto profesor-estudiante, durante el primer semestre del curso? También se caracterizó desde diferentes variables socio-educativas, el estudiantado que está cursando la carrera de profesorado de Biología en el año 2020.

\section{Metodología}

El diseño de esta investigación es de corte mixto y se corresponde con el enfoque de predominancia cuantitativa (modelo CUAN cual) que proponen Creswell y Plano Clark (2007).

El universo de análisis de la investigación lo conforman los estudiantes de la carrera de profesorado de Biología en uno de los Centros de Formación de Docentes del interior del país, así como también los profesores de las asignaturas específicas de dicha carrera en ese Instituto.

Para responder a las preguntas de investigación se diseñó el instrumento de autorreporte de encuesta digital. La encuesta en línea auto-administrada mediante Internet implica, por su modalidad, una mayor garantía de anonimato y una menor aquiescencia y deseabilidad social de las respuestas (McCullough, 1998; Heerwegh y Loosveldt, 2009). Se armaron dos cuestionarios por parte del equipo de investigación, uno se aplicó a estudiantes y el otro a profesores, me- diante Formulario de Google Drive durante el segundo semestre del año lectivo 2020, entre los meses de septiembre y octubre. El cuestionario tuvo preguntas con escala Likert, múltiple opción, opciones desplegables, casilla de verificación, cuadrícula de varias opciones y cuadrícula de casillas. Para varias de las preguntas se habilitó la opción de comentar, a través de la opción de respuesta de "párrafo".

Para los estudiantes, el acceso al marco muestral se dio a través de las clases presenciales que se tuvieron en las últimas semanas de la segunda mitad del año lectivo 2020. Los investigadores proporcionaron a los profesores participantes el link al formulario de la encuesta el que se administró, en casi todos los casos, en el horario de clase correspondiente a distintas asignaturas específicas de la carrera. Como el desarrollo de las clases se dio principalmente en modalidad on-line sincrónica, se hizo operativo la aplicación de la encuesta por diferentes vías: WhatsApp, a través de invitación por correo electrónico o a través de las aulas en plataforma CREA del CFE. Por otra parte, a los profesores se informó de la realización de la encuesta utilizando el grupo de trabajo que tiene el Departamento académico, grupo de WhatsApp; luego se envió a la casilla de correo personal el link al formulario. Este fue contestado por los profesores en el correr de los siguientes 3 días.

Las dimensiones y variables consideradas en el diseño de la encuesta utilizada para recoger información, son una adaptación a Uruguay de instrumentos que fueron ya validados en el contexto internacional (Marcelo \& Zapata, 2008; Cebreiro, Fernández \& Arribi, 2017). La Tabla N. ${ }^{\circ} 1$ presenta las mismas para la encuesta aplicada a estudiantes.

Tabla N. ${ }^{\circ}$ 1. Dimensiones y variables consideradas en la encuesta aplicada a estudiantes de la carrera de profesorado de Biología, año lectivo 2020

\begin{tabular}{|c|c|c|c|}
\hline Dimensiones & Sección de la Encuesta & $\begin{array}{c}\text { Preguntas de la } \\
\text { Encuesta }\end{array}$ & Variables \\
\hline $\begin{array}{c}\text { Perfil socio-demográfico de los estu- } \\
\text { diantes de la carrera de profesorado de } \\
\text { Biología. }\end{array}$ & $\begin{array}{c}\text { I } \\
\text { (Contextualización del en- } \\
\text { cuestado) }\end{array}$ & $\mathrm{N} .{ }^{\circ} 1,2,3,4$ y 5 & $\begin{array}{c}\text { Edad; sexo; año de ingreso a la carre- } \\
\text { ra; nivel que cursa; asignaturas espe- } \\
\text { cíficas que cursa. }\end{array}$ \\
\hline $\begin{array}{c}\text { Soporte tecnológico utilizado por es- } \\
\text { tudiantes para la consecución de los } \\
\text { cursos en el año 2020. }\end{array}$ & $\begin{array}{c}\text { II } \\
\text { (Recursos tecnológicos y } \\
\text { conectividad) }\end{array}$ & $\mathrm{N} .^{\circ} 6$ y 7 & $\begin{array}{c}\text { Dispositivo tecnológico; tipo de co- } \\
\text { nectividad utilizada. }\end{array}$ \\
\hline $\begin{array}{c}\text { Recursos digitales on-line utilizados } \\
\text { por profesores y estudiantes para de- } \\
\text { sarrollar procesos de enseñanza y de } \\
\text { aprendizaje }\end{array}$ & $\begin{array}{c}\text { Enseñanza y aprendizaje en } \\
\text { el 1 1er semestre) }\end{array}$ & $\begin{array}{c}\mathrm{N} .{ }^{\circ} 8,9,14,15,16,26 \text { y } 27 \\
16,17\end{array}$ & $\begin{array}{c}\text { Plataforma utilizada para construir el } \\
\text { aula virtual; herramienta digital para } \\
\text { la comunicación sincrónica y asincró- } \\
\text { nica; enseñanza on-line de activida- } \\
\text { des prácticas de laboratorio de las } \\
\text { Ciencias Biológicas. }\end{array}$ \\
\hline
\end{tabular}




\begin{tabular}{|c|c|c|c|}
\hline Dimensiones & Sección de la Encuesta & $\begin{array}{c}\text { Preguntas de la } \\
\text { Encuesta }\end{array}$ & Variables \\
\hline $\begin{array}{l}\text { Características de las aulas virtuales } \\
\text { utilizadas por el docente para el curso } \\
\text { on-line. }\end{array}$ & III & N. ${ }^{\circ} 20,21$, & $\begin{array}{c}\text { Organización de las aulas virtuales; } \\
\text { frecuencia de uso de las aulas virtua- } \\
\text { les. }\end{array}$ \\
\hline Perfil del docente en la virtualidad. & III & $\begin{array}{l}\text { N. }{ }^{\circ} 10,11,12 \\
13,18 \text { y } 19\end{array}$ & $\begin{array}{c}\text { Supervisión y feed-back del docente } \\
\text { en las aulas; manejo de los tiempos } \\
\text { pedagógicos. }\end{array}$ \\
\hline Características del b-learning. & $\begin{array}{c}\text { IV } \\
\left(\begin{array}{c}\text { Enseñanza y aprendizaje en } \\
\left.\text { el } 2^{\circ} \text { semestre }\right)\end{array}\right.\end{array}$ & N. ${ }^{\circ} 28$ y 29 & $\begin{array}{c}\text { Continuidad de la enseñanza; víncu- } \\
\text { los entre actividades presenciales- } \\
\text { actividades a distancia }\end{array}$ \\
\hline $\begin{array}{l}\text { Percepción de calidad educativa en el } \\
\text { cursado } 2020 .\end{array}$ & III y IV & $\begin{array}{l}\text { N. } 22,23,24 \\
25,30 \text { y } 31\end{array}$ & $\begin{array}{l}\text { Valoración del nivel de aprendizaje } \\
\text { durante } 1^{\text {er }} \text { semestre; valoración del } \\
\text { nivel de enseñanza durante } 1^{\text {er }} \text { se- } \\
\text { mestre; valoración del nivel de ense- } \\
\text { ñanza durante el } 2^{\circ} \text { semestre }\end{array}$ \\
\hline
\end{tabular}

Fuente: elaboración propia.

En la tabla $\mathrm{N} .^{\circ} 2$ presentamos las dimensiones y variables consideradas en el diseño de la encuesta utilizada para recoger información de los profesores formado- res que están a cargo de asignaturas específicas de la carrera de profesorado de Biología.

Tabla N. ${ }^{\circ}$ 2. Dimensiones y variables consideradas en la encuesta aplicada a profesores de asignaturas específicas de la carrera de profesorado de Biología, año lectivo 2020

\begin{tabular}{|c|c|c|c|}
\hline Dimensiones & Sección de la Encuesta & $\begin{array}{c}\text { Preguntas de la } \\
\text { Encuesta }\end{array}$ & Variables \\
\hline $\begin{array}{c}\text { Antigüedad docente en el sistema } \\
\text { formación de profesores. }\end{array}$ & $\begin{array}{c}\text { I (Contextualización del en- } \\
\text { cuestado) }\end{array}$ & N. ${ }^{\circ} 3$ & Años de antigüedad docente. \\
\hline $\begin{array}{c}\text { Soporte tecnológico utilizado por } \\
\text { profesores para el dictado de los } \\
\text { cursos en el año } 2020 .\end{array}$ & $\begin{array}{l}\text { II (Recursos tecnológicos y } \\
\text { conectividad) }\end{array}$ & N. ${ }^{\circ} 1$ y 2 & $\begin{array}{l}\text { Dispositivo tecnológico; tipo de conectivi- } \\
\text { dad utilizada. }\end{array}$ \\
\hline $\begin{array}{c}\text { Recursos tecnológicos utilizados } \\
\text { para el contacto profesor -alum- } \\
\text { no en el 1er semestre y continui- } \\
\text { dad semanal. }\end{array}$ & $\begin{array}{c}\text { III (Enseñanza y aprendizaje } \\
\text { en el 1er Semestre) }\end{array}$ & N. ${ }^{\circ} 4,5$ y 9 & $\begin{array}{l}\text { Plataforma utilizada para construir el aula } \\
\text { virtual; herramienta digital para la comuni- } \\
\text { cación sincrónica y asincrónica; enseñanza } \\
\text { on-line de actividades prácticas. Tiempo } \\
\text { semanal destinado al contacto virtual. }\end{array}$ \\
\hline \begin{tabular}{|c|}
$\begin{array}{c}\text { Uso de recursos y opciones plan- } \\
\text { teadas para el proceso de ense- } \\
\text { ñanza-aprendizaje }\end{array}$ \\
\end{tabular} & V & 7 & Valoración según frecuencia de uso \\
\hline $\begin{array}{c}\text { Opciones que reflejan presencia } \\
\text { y apoyo del docente, así como } \\
\text { también formas de evacuar dudas } \\
\text { a los estudiantes. }\end{array}$ & 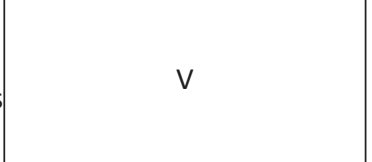 & 6 y 8 & $\begin{array}{c}\text { Supervisión e intercambio entre docente } \\
\text { y alumno a través de distintos medios vir- } \\
\text { tuales. }\end{array}$ \\
\hline \begin{tabular}{|c|} 
Evaluación del aprendizaje grupal \\
y el nivel global del curso.
\end{tabular} & V & 10 y 11 & $\begin{array}{l}\text { Valoración desde escaso a muy bueno y } \\
\text { desde muy bajo a excelente. }\end{array}$ \\
\hline $\begin{array}{l}\text { Cambios instrumentados por el } \\
\text { docente. }\end{array}$ & $\begin{array}{c}\text { IV (Enseñanza y aprendizaje } \\
\text { en el } 2 \text { do Semestre) }\end{array}$ & 1 & $\begin{array}{l}\text { Continuidad de la enseñanza; vínculos en- } \\
\text { tre actividades presenciales-actividades a } \\
\text { distancia }\end{array}$ \\
\hline
\end{tabular}




\section{Resultados}

Se recogieron 62 respuestas de los estudiantes, representando la muestra un 77,5 \% del estudiantado que cursó la carrera en 2020 . Tabla N. ${ }^{\circ} 3$.

Tabla N. ${ }^{\circ}$ 3. Características de la muestra estudiantil, año 2020

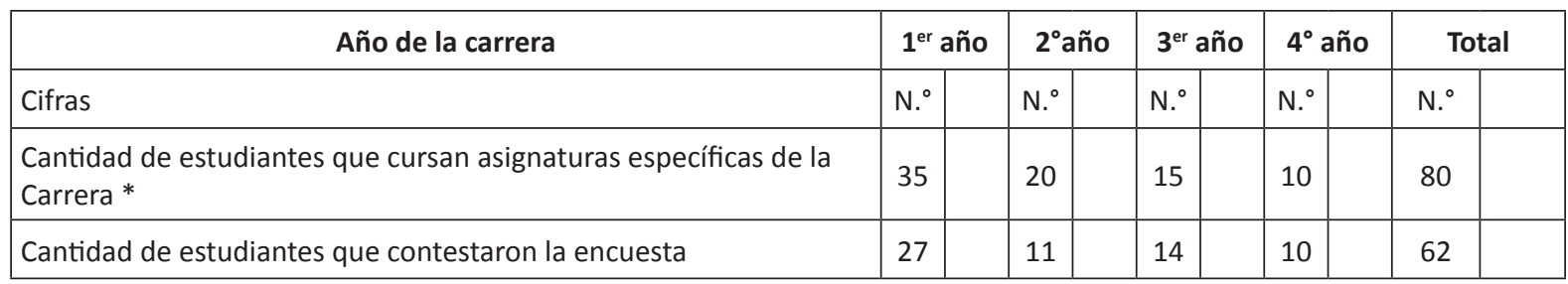

* Promedio de estudiantes matriculados en las distintas asignaturas específicas, para cada uno de los niveles. Fuente: elaboración propia

En la tabla $\mathrm{N} .^{\circ} 4$ vemos la muestra docente: 11 respuestas, representando un promedio general de $48 \%$, si cada asignatura de la carrera fuera dictada por un profesor diferente.

Tabla 4. Características de la muestra docente, año 2020

\begin{tabular}{|c|c|c|c|c|c|c|}
\hline Año de la carrera & $1^{\text {er }}$ & & $2^{\circ}$ & $3^{\text {er }}$ & $4^{\circ}$ & \\
\hline Cifras & $\mathrm{N} .^{\circ}$ & & N. ${ }^{\circ}$ & N. ${ }^{\circ}$ & N. ${ }^{\circ}$ & N. ${ }^{\circ}$ \\
\hline Cantidad de asignaturas específicas de la Carrera & 5 & & 5 & 7 & 6 & 23 \\
\hline Cantidad de profesores que contestaron la encuesta & 4 & & 3 & 2 & 2 & 11 \\
\hline
\end{tabular}

Fuente: elaboración propia

Ordenamos los resultados presentando gráficos y tablas que abordan el perfil socio-educativo de los estudiantes y la tecnología usada por profesores y estudiantes, para la consecución de los cursos. Se presentan los resultados de las 2 primeras dimensiones de la encuesta aplicada, tanto a estudiantes como a profesores (Tablas 1 y 2). Específicamente, se presentan y discuten resultados sobre: 1- edad del estudiantado; 2- año de ingreso a la formación docente; 3- número de asignaturas específicas que cursa en cada año de la carrera; 4- sexo del estudiantado; 5- dispositivos tecnológicos utilizados por profesores y estudiantes; 6- conectividad a Internet utilizada por profesores y estudiantes. 


\section{Edad del estudiantado que cursa la carrera de profesorado de Biología}

Para cada uno de los cuatro niveles de la carrera, mostramos los resultados en la Figura N. ${ }^{\circ} 1$.

Gráfico 1. Edad de los estudiantes en cada uno de los 4 niveles

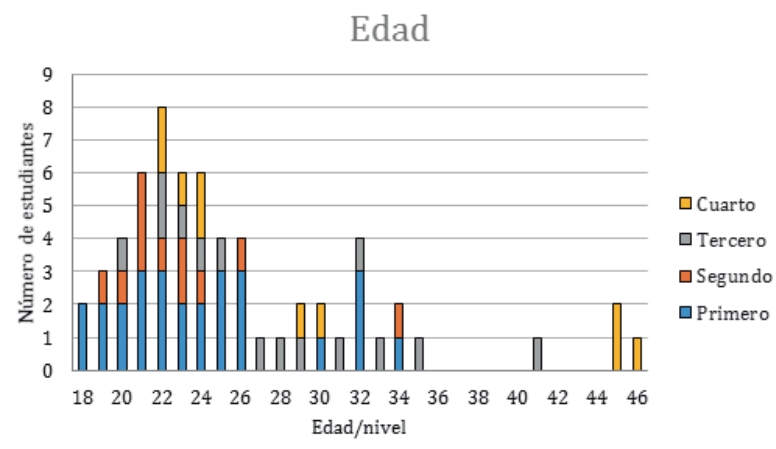

En el gráfico 1 vemos el perfil de edad en los 4 niveles de la carrera; la mayoría de los estudiantes están comprendidos entre los 18 y 26 años (69\%), siendo la edad de 22 la que presenta mayor cantidad de alumnos en el acumulado para los cuatro niveles (13\%) y 26 años el promedio general. Vemos que la edad 18, que corresponde a la de ingreso al nivel terciario para aquellos estudiantes que egresan del nivel medio en el tiempo esperado, solo se da en un número muy escaso: 2 estudiantes, un 7\%. La edad promedio de los estudiantes de cada nivel es de 24 años en primero, 23 en segundo, 29 en tercero y 31 en cuarto, lo que representa entre seis y diez años de rezago respecto a la edad esperada de egreso de educación media. El rezago que se viene dando en la culminación de la enseñanza media, sumado a la opción de ingresar a carreras universitarias y no a la docencia (INEEd, 2019), más el propio rezago que se da en la propia carrera de formación docente, explican estos resultados. Lejos estamos de tener mayoría de egresados de la formación inicial de profesores, a los 21 años, lo que correspondería si el ingreso a la carrera se diera a los 18 años y el trayecto por la formación docente se diera sin rezago. De hecho, en $4^{\circ}$ año no tenemos ningún estudiante con 21 años. Los resultados obtenidos son similares a los obtenidos en trabajos previos en Uruguay (INEEd, 2013).

\section{Año de ingreso a la carrera de profesorado de Biología}

El gráfico de barra número 2, muestra el año de ingreso a la carrera para los estudiantes de cada uno de los cuatro niveles.

Gráfico 2. Año de ingreso a la carrera en cada uno de los 4 niveles

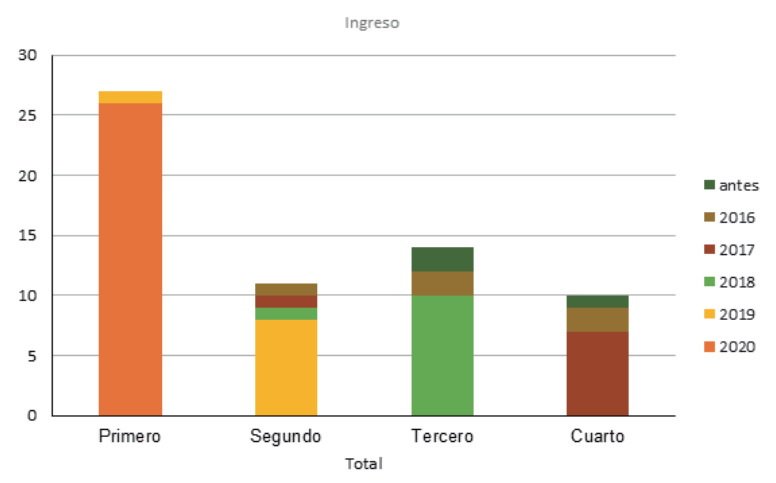

Si bien la mayoría de los cursantes en cada nivel ingresó a la carrera en el año que correspondía (los de $1^{\circ}$ en 2020 , los de $2^{\circ}$ en 2019 , los de $3^{\circ}$ en 2018 y los de $4^{\circ}$ en 2017), vemos que en todos los niveles hay un rezago importante: $4 \%$ en primer año, $27 \%$ en segundo, $29 \%$ en tercero, 30\% en cuarto. En promedio el rezago es de un $18 \%$, que aumenta a lo largo de la carrera, significativamente entre primer y segundo año.

\section{Asignaturas específicas de cada nivel que está cursando en el año 2020}

El gráfico 3 muestra, para asignaturas específicas de la carrera de profesorado de Biología, el año de la carrera que está transitando el estudiantado. La tabla 5 muestra la cantidad de asignaturas específicas que se están cursando en cada nivel. 
Gráfico 3. Asignaturas específicas de cada nivel, que se cursan en el año 2020

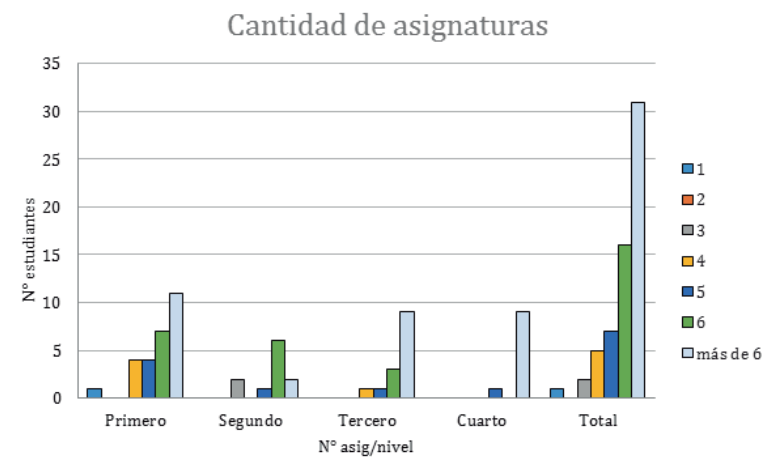

Vemos en el gráfico 3 que un porcentaje importante de estudiantes cursa asignaturas de más de un nivel: un $19 \%$ a nivel general. Los estudiantes, especialmente al llegar a $3^{\circ}$ y $4^{\circ}$, optan por volver a cursar asignaturas específicas de años anteriores en lugar de dar el examen para aprobarlas. Particularmente entre los estudiantes cuyo nivel más avanzado es tercer año, el $43 \%$ se encuentran en esa situación. En la tabla 5 vemos que un porcentaje alto de estudiantes $(50 \%)$, dice estar cursando más de 6 asignaturas específicas en cada nivel, número este que es superior al que corresponde a cualquier año de la carrera. El estudiante está optando por volver a cursar asignaturas antes que dar el examen reglamentado (quizá procurando la exoneración), o volver a cursarla al haber perdido la calidad de reglamentado, para no dar el examen (o no poder hacerlo por normativa vigente), en calidad de libre.

Tabla 5. Cantidad de asignaturas que cursa

\begin{tabular}{|c|c|c|}
\hline N. $^{\circ}$ asignaturas & Total & Porcentaje \\
\hline 1 & 1 & $2 \%$ \\
\hline 2 & 0 & $0 \%$ \\
\hline 3 & 2 & $3 \%$ \\
\hline 4 & 5 & $8 \%$ \\
\hline 5 & 7 & $11 \%$ \\
\hline 6 & 16 & $26 \%$ \\
\hline Más de 6 & 31 & $50 \%$ \\
\hline
\end{tabular}

El rezago se correlaciona con la tendencia constatable: volver a cursar asignaturas de años anteriores (gráfico 3 )

\section{Sexo del estudiantado}

En el gráfico 4 mostramos las respuestas de los estudiantes con respecto a su identificación sexual.

Gráfico 4. Sexo del estudiantado en cada uno de los 4 niveles

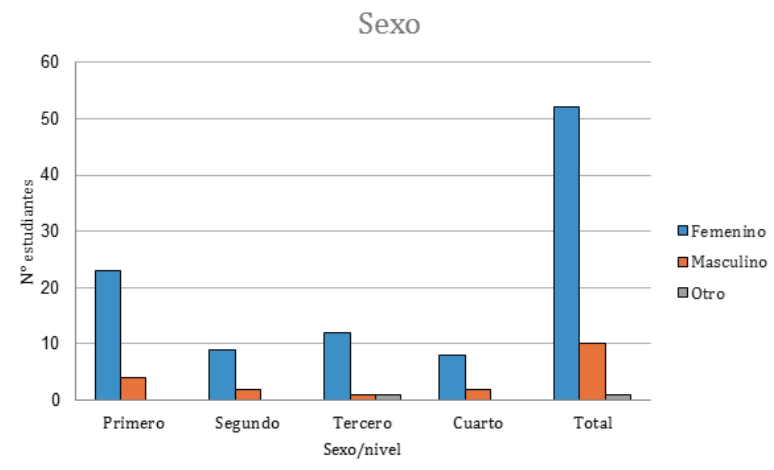

Vemos que en cada uno de los 4 niveles hay una clara primacía de mujeres sobre varones, siendo el acumulado de 52 personas contra 10 (índice de feminización de 5,2). Solo 1 estudiante no se identifica con la clasificación binaria clásica de los sexos, marcando la opción "otro". La feminización de la formación docente es también una constatación confirmada por otros estudios realizados en la formación docente; en Uruguay la feminización del estudiantado es también muy alta (IF=3,4; a partir de datos de INEEd, 2019), aunque no tanta como en nuestra población de estudio.

\section{Dispositivos tecnológicos utilizados para la enseńanza y aprendizaje a distancia, de las asignaturas específicas de la carrera de profesorado}

En las tablas 6 y 7 y el gráfico 5 se muestran los resultados sobre el uso dado por los estudiantes y por los profesores, a distintos dispositivos tecnológicos que posibilitaron mantener contacto con los cursos de las asignaturas específicas durante el primer semestre (en modalidad a distancia) y durante el segundo semestre (en modalidad b-learnig) del año lectivo 2020. Se tuvo en cuenta el total de respuestas y no de estudiantes, porque un estudiante pudo haber señalado más de un recurso. La pregunta formulada a estudiantes y docentes fue: "Señale para cada recurso tecnológico el uso que en su caso tuvo, a los efectos de mantener contac- 
to con los cursos de profesorado del Instituto". En una cuadrícula de casillas, se presentaban distintos dispositivos informáticos y las opciones a marcar para cada uno ellos: ningún uso, uso escaso, uso frecuente y uso muy frecuente. Particularmente, la tabla 6 y el gráfico
5 se presentan cuáles fueron los recursos tecnológicos considerados como de uso muy frecuente por los estudiantes, mientras que en la tabla 7 se registra lo mismo para los profesores.

Tabla 6. Recursos tecnológicos señalados como de uso muy frecuente por los estudiantes

\begin{tabular}{|c|c|c|c|c|c|c|}
\hline $\begin{array}{c}\text { Dispositivo } \\
\text { utilizado }\end{array}$ & 1er Año & $2^{\circ}$ Año & 3er Año & $4^{\circ}$ Año & $\begin{array}{c}\text { Total de } \\
\text { respuestas }\end{array}$ & $\begin{array}{c}\text { Porcentaje } \\
\text { de respuestas }\end{array}$ \\
\hline PC & 1 & 2 & 1 & 0 & 4 & 5 \\
\hline XO & 2 & 1 & 5 & 5 & 13 & 17 \\
\hline Laptop & 16 & 4 & 9 & 5 & 34 & 45 \\
\hline Tablet & 0 & 0 & 0 & 0 & 0 & 0 \\
\hline Celular & 12 & 3 & 7 & 3 & 25 & 33 \\
\hline Total & 31 & 10 & 22 & 13 & 76 & 100 \\
\hline
\end{tabular}

Gráfico 5. Recursos informáticos de uso muy frecuente.

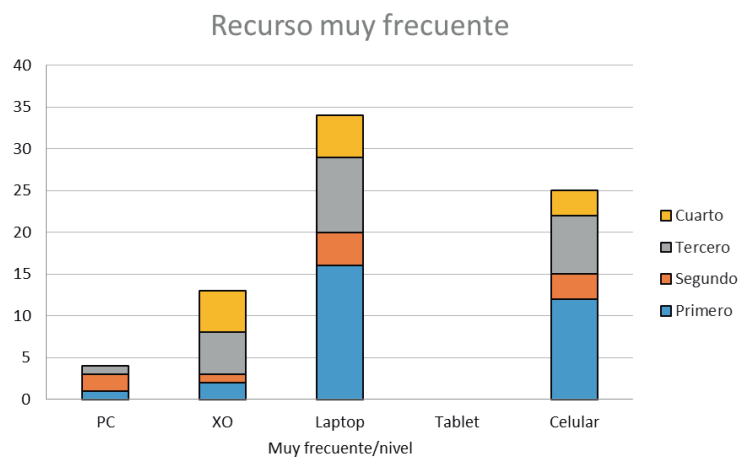

Vemos claramente que las laptops personales (45\%) y el celular (33\%), son los recursos tecnológicos señalados como los más frecuentemente usados por los estudiantes. Ambos dispositivos móviles son el recurso tecnológico más utilizado - por los estudiantes en todos los niveles de cursado - para mantener contacto con los cursos de las asignaturas específicas. Las laptops de Plan Ceibal (XO), han sido solo para una cantidad reducida de alumnos el recurso de uso muy frecuente, $17 \%$ del total de respuestas. El uso preferente de las XO aumenta a un $28,5 \%$ (10 respuestas que marcaron la opción XO en esos dos niveles, para un total de 35 ) entre estudiantes de tercer y cuarto año, igualado al uso del celular,

Los resultados obtenidos sobre el uso dado a las laptops XO en la formación de profesores, es coincidente con otras investigaciones que se han realizado en formación docente en los últimos años (Rodríguez pero inferior a la laptop (40\%). Este dato es importante, ya que es en 3er año de la carrera que los estudiantes reciben una laptop por parte del CFE.

Para los docentes formadores de las asignaturas específicas, el resultado es más contundente: tabla 7. Las laptops personales son el dispositivo tecnológico más usado para mantener contacto con los cursos de profesorado del Instituto $(52,7 \%)$, seguido del celular $(42,1 \%)$. Las XO no representó para ningún profesor el dispositivo tecnológico principal, explicable en parte porque muchos de ellos no cuentan con una XO operativa.

Tabla 7. Recursos tecnológicos señalados como de uso muy frecuente por los profesores

\begin{tabular}{|c|c|c|}
\hline Dispositivo utilizado & Respuestas & $\begin{array}{c}\text { Porcentaje de } \\
\text { respuestas }\end{array}$ \\
\hline PC & 1 & 5 \\
\hline XO & 0 & 0 \\
\hline Laptop & 10 & 53 \\
\hline Tablet & 0 & 0 \\
\hline Celular & 8 & 42 \\
\hline Total & 19 & 100 \\
\hline
\end{tabular}

Fuente: elaboración propia.

et al., 2019; Rodríguez y Grilli, 2018). Todas señalan que, tanto para estudiantes de formación docente como para profesores, las XO no son el principal recurso tecnológico para los cursos, siendo claramente las 
laptops personales las que ocupan el primer lugar. Algo similar se detectó también en el sistema educativo medio (Antelo, Bernasconi y Rodríguez, 2015). Las capacidades operativas del recurso (memoria, velocidad del procesador, sistema operativo y softwares instalados), y las condiciones de funcionamiento de las XO cuando el estudiante llega a formación docente, son seguramente variables que explican estos resultados que se vienen dando

\section{Conectividad a Internet utilizada por profesores y estudiantes}

Las tablas siguientes muestran los resultados para la opción "muy frecuente", a la pregunta: "Señale para cada tipo de conectividad el uso que en su caso tuvo, a los efectos de mantener contacto con los cursos de profesorado del Instituto", formulada tanto a estudiantes (tabla 8) como profesores de asignaturas específicas (tabla 9), en los cuatro niveles de la carrera.

Tabla 8. Conectividad usada de manera muy frecuente por los estudiantes para mantener contacto con los cursos de las asignaturas específicas de la carrera de profesorado de Biología

\begin{tabular}{|c|c|c|c|c|c|c|}
\hline Conexión & 1er Año & $\mathbf{2}^{\circ}$ Año & 3er Año & 4 $^{\circ}$ Año & Total de respuestas & Porcentaje de respuestas \\
\hline Ceibal hogar & 0 & 0 & 0 & 0 & 0 & 0 \\
\hline Ceibal educativa & 1 & 1 & 1 & 3 & 6 & 65 \\
\hline Wifi hogar & 18 & 6 & 14 & 10 & 2 & 3 \\
\hline Wifi vecino & 1 & 1 & 0 & 0 & 18 & 24 \\
\hline Móvil & 11 & 2 & 5 & 0 & 74 & 100 \\
\hline Total & 31 & 10 & 20 & 13 & & 78 \\
\hline
\end{tabular}

El $65 \%$ de las respuestas de los estudiantes señalan que la conectividad en su hogar a través del wifi personal, es la de uso más frecuente para realizar el cursado. En el profesorado la cifra porcentual es también alta, $58 \%$ (tabla 9). En segundo lugar, está la conectividad por internet móvil: $24 \%$ (estudiantes) y $26 \%$ (profesores). A medida que se avanza en la carrera, se nota en los estudiantes un mayor uso de la conectividad por wifi en el hogar, por sobre las demás opciones: $3^{\circ}$ y $4^{\circ}$ $24 / 33=73 \% ; 1^{\circ}$ y $2^{\circ} 24 / 41=58 \%$.

Tabla 9. Conectividad utilizada de manera muy frecuente por los docentes para mantener contacto con los cursos

\begin{tabular}{|c|c|c|}
\hline Conexión & Respuestas & $\begin{array}{c}\text { Porcentaje de } \\
\text { respuestas }\end{array}$ \\
\hline Ceibal hogar & 1 & 5 \\
\hline Ceibal educativa & 2 & 10 \\
\hline Wifi hogar & 11 & 58 \\
\hline Wifi vecino & 0 & 0 \\
\hline Móvil & 5 & 26 \\
\hline Total & 19 & 100 \\
\hline
\end{tabular}

La conectividad de Ceibal en los centros representó solo un $8 \%$, para estudiantes y un $10,4 \%$ para docentes, como de uso muy frecuente para la consecución de los cursos. También aquí los resultados obtenidos son coincidentes con otras investigaciones precedentes (Rodríguez y Grilli, 2018; Rodríguez et al., 2019). El confinamiento de las personas en sus casas, sumado al área de cobertura de la red Ceibal a instituciones educativas, son factores decisivos para este resultado constatado en este año de pandemia.

Resumiendo: las laptops personales y la conectividad a internet por wifi de los hogares, fueron los principales recursos, tanto en estudiantes como docentes, para mantener el contacto educativo de los cursos correspondientes a las asignaturas específicas de la carrera de profesorado, en el año 2020.

\section{Discusión de resultados}

La educación remota de emergencia que se dio en el año 2020, a consecuencia de la pandemia por la Covid19 , puso a prueba la capacidad de adaptación del sistema educativo. La circunstancia sirvió para mostrar que aspectos de la infraestructura y organización existente 
para el desarrollo de cursos en modalidad presencialidad, fueron relevantes y/o valiosos para la educación remota de emergencia.

Los resultados de la investigación en torno al objetivo de determinar los recursos tecnológicos que se utilizaron para los procesos de enseñanza-aprendizaje, en las distintas asignaturas específicas del profesorado de Biología en uno de los Institutos que forma profesores de Biología, confirma una tendencia constatada en otras investigaciones realizadas en el sistema de formación docente, previo al año 2020.

En un año educativo especial para Uruguay y para el mundo, como resultado de la pandemia por la Covid-19, en lo que respecta al uso de tecnologías que hicieron posible la consecución de los cursos en modalidad a distancia en el primer semestre y modalidad combinada en el segundo semestre, la investigación realizada confirma la relevancia que están teniendo los dispositivos portátiles: laptop y celulares. Son los dispositivos móviles personales, tanto en estudiantes como en profesores, los que han tenido mayor relevancia para mediar en los procesos educativos.

Las computadoras de Plan Ceibal entregadas a estudiantes en la educación media, estudiantes de $3^{\circ}$ de profesorado y profesores formadores, no son la principal herramienta o soporte tecnológico para mantener el cursado de las asignaturas específicas de la carrera de profesorado (aunque su uso sea similar a las laptop y celulares entre estudiantes de $3^{\circ}$ y $4^{\circ}$ año). Seguramente las siguientes son explicaciones válidas para este hecho constatado: capacidad del procesador, memorias del ordenador, softwares instalados, condiciones de funcionamiento del dispositivo móvil que fue entregado.

Con respecto a la conectividad, el estudio realizado muestra que la red personal en cada hogar fue la principal vía para hacer posible el contacto entre profesores y alumnos de la carrera de profesorado, tanto en el primer semestre como el segundo del año 2020.

En lo que respecta al perfil socio-educativo de los estudiantes, el estudio confirma también algunas tendencias constatadas por otros estudios, al tiempo que evidencia algunas otras. La mayoría de nuestro estudiantado tiene una edad por encima de lo esperado, tanto en el ingreso a la carrera como en el egreso. Tenemos por tanto un estudiantado que ingresa con una edad por encima de la esperable para el egreso del sistema educativo medio, a consecuencia de un retraso de egreso de dicho sistema, por dejar de estudiar después de hacerlo o porque se hace un tránsito por otros cursos terciarios, antes de ingresar a la carrera docente.
La feminización de la docencia viene siendo constatada desde hace tiempo en Uruguay. El INEEd presenta un índice de feminización de 3,4, siendo el obtenido en nuestra investigación, para la carrera de profesorado de Biología, de 5,2.

Finalmente, el estudio realizado muestra la tendencia en el estudiantado a recursar asignaturas de años previos. Un número importante de estudiantes de profesorado de Biología está cursando, en el año 2020, asignaturas específicas de más de un nivel de la carrera. Esta característica preocupa a docentes de asignaturas de Didáctica II y III ( $3^{\circ}$ y $4^{\circ}$ respectivamente) ya que implica la realización de una práctica docente pre-profesional con carencias conceptuales importantes, producto de que el estudiantado no tiene aprobado varias asignaturas específicas de los años precedentes.

\section{Conclusiones}

En los tiempos actuales enseñar y aprender ciencias implica un desafío tanto para docentes como para estudiantes ya que la inclusión de tecnologías toma un rol protagónico. Esto conlleva a repensar contenidos, metodologías, evaluaciones y finalidad de lo que se enseña. En este trabajo hemos constatado que dispositivos tecnológicos y que conectividad fueron las más relevantes para la enseñanza de las ciencias Biológicas en la formación de un profesor. Es importante también analizar el uso dado a las aulas virtuales en la plataforma CREA de Ceibal y las instancias sincrónicas y asincrónicas que se dieron en los procesos educativos, temas que también fueron investigados y que presentaremos en otro artículo.

La investigación realizada evidencia la necesidad de seguir mejorando y ajustando los servicios que brinda Ceibal a los Institutos de formación de profesores. Las condiciones que impuso la pandemia sobre el sistema educativo, y en particular sobre la formación docente, mostraron la real utilidad de los equipos informáticos y de la conectividad Ceibal, en los actores educativos: estudiantes y profesores. Más allá de la educación que debió darse en forma remota por la emergencia sanitaria, es claro que las plataformas educativas como la que proporciona Ceibal (Crea), seguirán siendo un potente recurso para las modalidades educativas a distancia y también aquellas que combinen presencialidad con virtualidad (b-learning), siendo básico y fundamental en ellas la disponibilidad de equipos informáticos y de una buena conectividad. 


\section{Referencias}

ANEP (2020). En el marco de la nueva presencialidad: Desafíos de la educación a distancia estrategias $y$ herramientas para docentes y comunidades educativas. ANEP-Plan Ceibal. https://www.anep. edu.uy/sites/default/files/images/2020/noticias/ julio/200703/Desafi\%CC\%81os\%20de\%20la\%20 educacio\%CC\%81n\%20a\%20distancia\%202020.pdf

Ángel Rueda, C, Valdés Godínesa, J., y Guzmán Flores T. (2017). Límites, desafíos y oportunidades para enseñar en los mundos virtuales. Innovación Educativa, 17(75), 149-168. http://www.scielo. org. $\mathrm{mx} /$ scielo.php?script=sci_arttext\&pid =S1665-26732017000300149

Antelo, A. M., Bernasconi, G., y Rodríguez Zidán, E. (2015). El Plan Ceibal en Uruguay y los desafíos del Modelo 1 a1. Estudio de las percepciones docentes sobre las prácticas de enseñanza y el uso pedagógico de las computadoras personales en la clase de Matemáticas. CBIE-LACLO 2015, Anais dos Workshops do IV Congresso Brasileiro de Informática na Educação (CBIE 2015). https:// www.researchgate.net/publication/300237145 El_Plan_Ceibal_en_Uruguay_y_los_desafios_del_ Modelo_1_a1_Estudio_de_las_percepciones_ docentes_sobre_las_practicas_de_ensenanza_y_ el_uso_pedagogico_de_las_computadoras_ personales_en_la_clase_de_Matemat

Aragay, X. (2020). Enseñanza remota de emergencia. Avanzar para no volver atrás. https://xavieraragay. com/eshoradetransformar/avanzar-para-novolver-atras?utm_source $=$ Contactes + Riedul $\mathrm{ab}+$ Castellano\&utm_campaign $=\mathrm{fce} 8 \mathrm{f} 37 \mathrm{ed} 2$ RSS_EMAIL_CAMPAIGN_CAST\&utm medium =email \&utm_term $=0-506$ e $7118 \mathrm{bd}$ fce8f37ed2-127456449

Area, M., San Nicolás, M., y Sanabria, A. (2018). Las aulas virtuales en la docencia de una universidad presencial: la visión del alumnado. RIED. Revista Iberoamericana de Educación a Distancia, 21(2), 179-198. http://revistas.uned.es/index.php/ried/ article/view/20666

Arranz, V., Aguado, D., y Lucía, B. (2008). La influencia del tutor en el seguimiento de programas eLearning. Estudio de acciones en un caso práctico. Revista de Psicología del Trabajo y de las Organizaciones, 24(1), 5-23. http://scielo. isciii.es/scielo.php?script=sci_arttext\&pid =S1576-59622008000100001
Cebreiro, B.; Fernández, C., y Arribi, J. (2017). Formación profesional a distancia: corriendo en la dirección equivocada. Píxel-Bit. Revista de Medios y Educación, (59), 65-76. https://dialnet.unirioja. es/servlet/articulo?codigo $=5769002$

Conde, A.; González, M., y Villagrán, A. (2018). Estudio sobre la modalidad de formación semipresencial del Consejo de formación en Educación (CFE). Informe 2017. Uruguay: ANEP-CFE. http:// repositorio.cfe.edu.uy/handle/123456789/153

Creswell, J., y Plano Clark, V. (2007). Designing and conducting Mixed Methods research. Thousand Oaks, CA: Sage. DOI:10.1177/1558689807306132

Echeverría Samanes, B. (2002). Gestión de la competencia de Acción Profesional. Revista De Investigación Educativa, 20(1), 7-43. https:// revistas.um.es/rie/article/view/97411

García Aretio, L. (2014). Bases, mediaciones y futuro de la educación a distancia en la sociedad digital. Editorial Síntesis.

Heerwegh, D., y Loosveldt, G. (2009). Explaining the intention to participate in a web survey: a test of theory of planned behavior. International Journal of Social Research methodology, 12, 181-195. https:// www.researchgate.net/publication/233229597_ Explaining_the_intention_to_participate_in_a_ web_survey_A_test_of_the_theory_of_planned_ behaviour

Hidalgo, S., Orozco, M., y Daza, M. (2015). Trabajando con Aprendizaje Ubicuo en los alumnos que cursan la materia de Tecnologías de la Información. Revista Iberoamericana para la Investigación y el Desarrollo Educativo, 6(11), https://dialnet. unirioja.es/servlet/articulo?codigo $=5280203$

Inciarte Rodríguez, M. (2008). Competencias docentes ante la virtualidad de la educación superior. Revista Télématique, (7)2, 2008, 19-38. https:// www.redalyc.org/pdf/784/78470202.pdf

INEEd. (2013). Evolución de las profesiones docentes en Uruguay. Desafíos para la próxima década. Montevideo. INEEd. https://www.ineed.edu.uy/ images/pdf/evolucion-profesiones-docentes.pdf

INEEd. (2019). Informe sobre el estado de la educación en Uruguay 2017-2018. Montevideo: INEEd. https://www.ineed.edu.uy/images/ ieeuy/2017-2018/pdf/Informe-sobre-el-estadode-la-educacion-en-Uruguay-2017-2018.pdf 
Marcelo, C., y Zapata, M. (2008). Cuestionario para la evaluación: Evaluación de la calidad para programas completos de formación docente a través de estrategias de aprendizaje abierto y a distancia. Metodología de uso y descripción de indicadores. RED, Revista de Educación a Distancia. Número monográfico VII.- 30 de diciembre de 2008. Número especial dedicado a la evaluación de la calidad en entornos virtuales de aprendizaje. https://revistas.um.es/red/article/view/125191

McCullough, D. (1998). Web-based market research ushers in new age. Marketing methodology, 12, 181-195.

Moore, M. G., y Kearsley, G. (2005). Distance education: A system view (2a. ed.). Belmont, CA, EE. UU.: Wadsworth Publishing.

Pascoal, D. (2016). Hacia el aprendizaje virtual. Revista Multidisciplinaria Dialógica, (13)2, 103-116. https://dialnet.unirioja.es/servlet/ articulo?codigo $=6219287$

Rodríguez Zidán, E., Yot, C., Cabrera, C., Zorrilla, J., y Grilli Silva, J. (2019). Desafíos para el diseño de nuevas pedagogías basadas en tecnologías móviles. Cadernos de Pesquisa, Sao Paulo, (49)172, 236-259. https://www.scielo.br/scielo. php?pid=S0100-15742019000200236\&script=sci_ arttext\&tlng=es

Rodríguez, E., y Grilli Silva, J (2018). El b-learning en la educación terciaria. Estudio de las percepciones de docentes y estudiantes sobre la apropiación y el uso de las plataformas digitales en la formación inicial de profesores. ANEP-CFE. http://repositorio. cfe.edu.uy/handle/123456789/486

Vanden Berg, L. (2015). Profesorado Semi-presencial en Uruguay: Enfoque de los docentes de las asignaturas específicas de Biología, Física y Química y recursos didácticos elegidos para trabajar en la modalidad. Montevideo, Universidad ORT. https:// sisbibliotecas.ort.edu.uy/cgi-bin/koha/opacdetail.pl?biblionumber $=81814$

Yot, C., y Marcelo, C (2013). Tareas y competencias del tutor on-line. Profesorado. Revista del currículum y formación del profesorado, 17(2), 305-325. https://www.ugr.es/ recfpro/rev172COL5.pdf 